\title{
SÍNDROME DE DOWN POR MOSAICO, REPORTE DE CASO ECUADOR.
}

\author{
MOSAIC DOWN SYNDROME, CASE REPORT ECUADOR. \\ Cristian Carlos Ramírez Portilla1, Michelle Domenica Sarmiento Rubio², Mónica Carolina \\ Quezada Pardó, Jessica Tamara Orellana Córdova².
}

\section{RESUMEN}

El síndrome de Down, es una de las principales causas de discapacitada intelectual relacionada a alteraciones en los cromosomas humanos, el síndrome de Down por mosaísmo es uno de los tres tipos de alteración cromosómica que se presentan en estos pacientes, encontrándose en $1 \%$ al $4 \%$ de los casos reportados de este síndrome, en los cuales las características físicas son casi imperceptibles a una simple valoración. Presentamos el caso clínico de paciente masculino de 9 meses de edad que acudió a valoración médica por retraso leve madurativo e infecciones respiratorias a repetición.

\section{ABSTRACT}

Down syndrome is one of the main causes of intellectual disability related to alterations in human chromosomes. Mosaic Down syndrome is one of the three types of chromosomal alteration that occurs in these patients, being $1 \%$ at $4 \%$ of the reported cases of this syndrome, in any of the physical characteristics are almost imperceptible to a simple assessment. We present the clinical case of a 9-month-old male patient who attended a medical evaluation for mild maturational delay and repeated respiratory infections.

\section{INTRODUCCIÓN}

El síndrome de Down fue descrito por

Eprimera vez por el medico británico John Langdon Haydon Down en el año 1866 que describe las características físicas de varias personas con discapacidad intelectual en las cuales no se conocía su etiología. En el año 1958 Jérôme Lejeune genetista medico realiza varias investigaciones en los cromosomas humanos de personas con discapacidad intelectual y características físicas semejantes, donde se pudo observar alteraciones en el par 21 de los cromosomas de estas personas afectadas con este síndrome ${ }^{1}$.

El síndrome se ha observado que presenta una incidencia de 1 por cada 700 nacidos en América del Sur, siendo el síndrome de Down por mosaico el $1 \%$ al $4 \%$ de la causa etiológica cromosómica de esta patología, observándose la configuración de los cromosomas de la siguiente manera 47, XX+21/46, XX (pacientes femeninas), 47, $X Y+21 / 46, X Y$ (pacientes masculinos) ${ }^{2}$.

Dos son las causas principales de la aparición de síndrome de Down por mosaico, siendo la primera por falta de división meiótica, sobre todo observada en mujeres de edad mayor a los 37 años, produciendo una mala división de los óvulos en el momento de la concepción y así ocurriendo una línea celular con mayor cantidad de cromosomas que otra; y la segunda causa es de origen mitótico en la cual la concepción es totalmente normal pero a medida que se van dividiendo las células en algún momento aumentó la cantidad de cromosoma en una línea celular y en otras se mantiene normales ${ }^{3}$.

\section{PRESENTACIÓN DEL CASO}

Paciente masculino, producto del tercer embarazo, precedido de dos abortos espontáneos de una pareja. Al momento de su nacimiento, la madre tenía 34 años y el padre 35 años. El embarazo del propósito fue controlado, presentando infección de tracto urinario al quinto mes y polihidramnios al octavo. El nacimiento fue por cesárea debido a cesárea anterior; a través de la prueba de Capurro se estimó: 39 semanas de gestación, el conteo APGAR fue: 8 al minuto y 9 a los cinco

\begin{tabular}{l}
\hline Máster en genètica, \\
especialista en biología \\
molecular, docente Universidad \\
Catòlica de Cuenca, Ecuador \\
\hline 2 Médico general en funciones \\
hospitalarias MSP, Ecuador.
\end{tabular}

Correspondencia a: Cristian Carlos Ramírez Portilla Correo electrónico: ctaramirez66@hotmail.com

Telefono: 593984874713 ORCID: https://orcid.org/00000002-1668-1192

Palabras clave: Síndrome de Down, mosaico, trisomía 21, trastorno de los cromosomas.

Keywords: Down syndrome, mosaic, trisomy 21, chromosome disorders.

Procedencia y arbitraje: no comisionado, sometido a arbitraje externo.

Recibido para publicación: 6 de mayo de 2020 Aceptado para publicación: 8 de diciembre de 2020

Citar como:

Ramírez Portilla CC, Sarmiento Rubio MD, Quezada Pardo MC, Orellana Córdova JT. Síndrome de down por mosaico, reporte de caso ecuador. Rev Cient Cienc Med. 2020; 23(2): 267-270 
minutos, peso: $3400 \mathrm{~g}$, talla: $48 \mathrm{~cm}$, perímetro cefálico: $38 \mathrm{~cm}$, perímetro torácico: $33 \mathrm{~cm}$; fue dado de alta hospitalaria a las 48 horas de vida.

Al tercer mes de nacido es remitido al servicio de pediatría por presentar hipertermia $\left(38^{\circ} \mathrm{C}\right)$, tos productiva, rinorrea bilateral amarillenta y acrocianosis s; es hospitalizado por ocho días con tratamiento de antibióticos (ampicilina y gentamicina); y dado de alta con diagnóstico de neumonía bacteriana, según consta en informes previos archivados en la historia clínica de la paciente. Al sexto mes de edad acude nuevamente a consulta externa por presentar hipertermia (38.5 C) rinorrea bilateral transparente, tos no productiva y sostén cefálico disminuido, motivo por lo cual se administra antibioticoterapia mas expectorantes vía oral por 7 días por diagnóstico de bronconeumonía y se solicita valoración psicológica de test de DENVER, el cual presenta una puntuación del $75 \%$, correlacionándose este por debajo de la media para niños de su misma edad. Al noveno mes de edad se logra la atención por servicio de genética para valoración de retazo madurativo y cognitivo, en el cual luego de su valoración física observa pequeñas alteraciones faciales

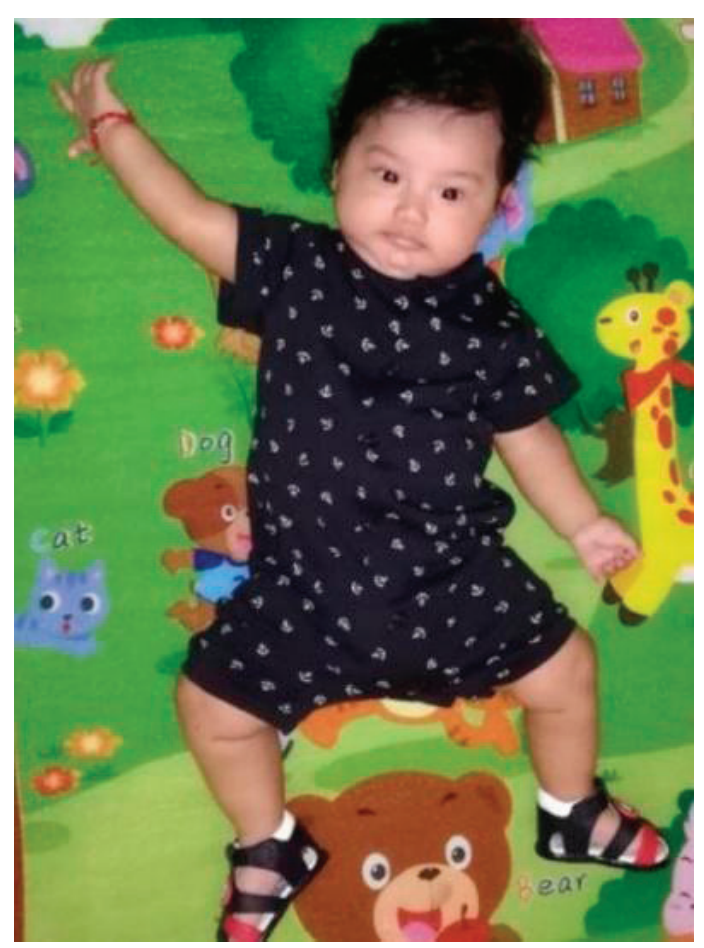

Figura 1: Paciente con sindrome de Down por mosaico, características clínicas leves como son: frente amplia, hipertelorismo leve, moderado epicanto bilateral y cuello corto con moderado sostén cefálico (Figura 1); se solicitó exámenes de cariotipo numérico el cual reporta después de un mes los siguientes resultados: 47, XY+21/ 46, XY, (Figura 2 y 3) siendo diagnosticado con Síndrome de Down por mosaico (Figura 4). Se solicitó interconsulta a psicología para valoración psicológica-afectiva-familiar para aceptación de este síndrome en un miembro de la familia, ya que los padres desconocían la presencia de esta patología en su hijo; De igual manera se solicitó valoración por fisiatría, seguimiento trimestral por pediatría y genética de esta casa de salud.

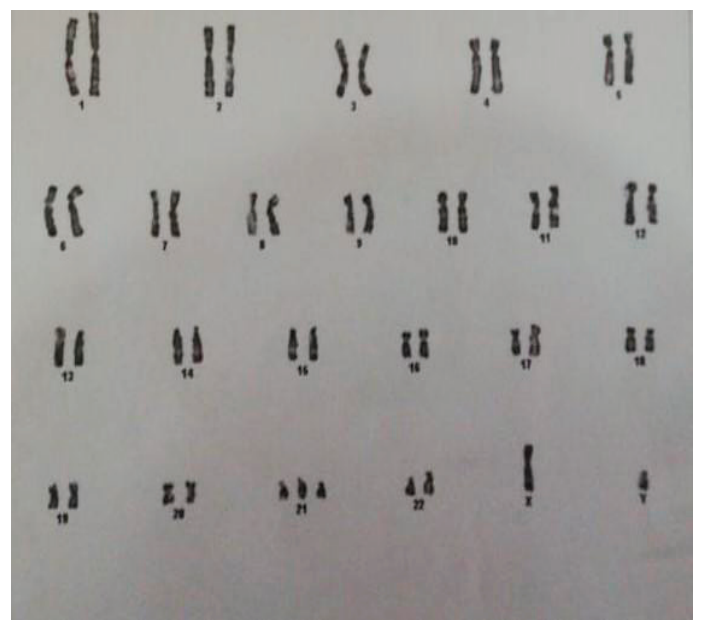

Figura 2: Cariotipo de paciente con síndrome de Down con presencia de trisomía 21 en 4 metafases.

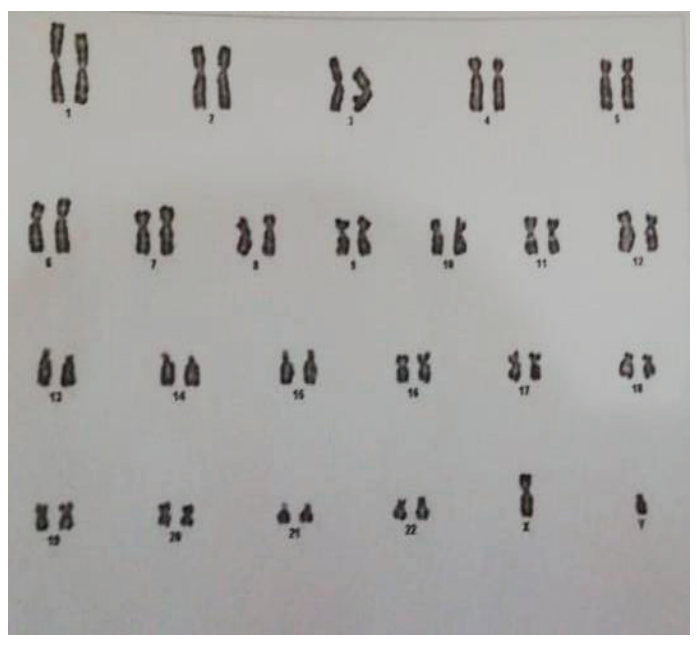

Figura 3: Cariotipo de paciente con sindrome de Down con presencia normal de cromosomas, realizadas en 26 metafases. 


\section{Laboratorio de citogenética}

\section{Cariotipo en sangre periférica.}

Se ha efectuado el cultivo de sangre periférica y realizado el recuento de 30

metafases, de los cuales 26 presentan 46 cromosomas y 4 presentan 47 cromosomas

Con la técnica de bandas $\mathrm{G}_{\text {a }}$ además de las células normales se observa en un $13 \%$ un

cromosomas 21 adicional.

Esta alteración está asociada con Síndrome de Down en mosaico.

CARIOTIPO: $46, \mathrm{XY}(26) / 47, \mathrm{XY},+21(4)$

Figura 4: Resultado escrito enviado por el laboratorio de citogenética donde describe la presencia de células en mosaico en este paciente.

\section{DISCUSIÓN}

Según la investigación realizada en el año 2013 por médicos pertenecientes a la Universidad Autónoma de México del Servicio de Genética del Hospital General de México Dr. Eduardo Liceaga, se puede observar que el porcentaje de personas con síndrome de Down por mosaico es baja como lo explicada en nuestro reporte de caso clínico, representando el $1 \%$ al $4 \%$ de todos los casos reportados de pacientes con síndrome de Down ${ }^{4}$.

De igual manera podemos observar que este paciente presenta rasgos físicos no muy notablesparasíndromedeDownydiscapacidad intelectual leve, lo mismo que es reportado en el año 2010 por en un estudio en el cual se relaciona las características citogenéticas del mosaico con las características clínicas fenotípicas e intelectuales del paciente, en el que se observó que en dependencia del porcentaje de células mosaico estén presente se relaciona directamente a su condición física e intelectual ${ }^{5}$.

Normalmente las personas con síndrome de Down por trisomía 21 presentan alteraciones faciales características como son frente amplia, epicanto bilateral, hipertelorismo, nariz en silla de montar, filtrum labial alargado, glosoptosis, labio inferior evertido, cuello corto, cardiopatías, alteraciones respiratorias y endocrinas ${ }^{6,7}$.

El diagnóstico preciso del síndrome de Down por mosaico se lo debe realizar mediante la recolección de sangre periférica, la cual se somete a un proceso de incubación para poder observar las diferentes etapas de la mitosis celular y una vez que se pueda observar la metafase se detiene esta incubación con reactivos específicos para poder observar con mayor facilidad estos cromosomas los cuales deben ser observados en un mínimo de 30 metafases y encontrándose en los pacientes con SD por mosaico células con tres cromosomas 21 y otras células con dos cromosomas $21^{8}$.

En general cuando se realice el diagnóstico de SD sea clínicamente o por medio del cariotipo es ideal informar a los progenitores sobre la condición de su hijo, motivo por lo cual se sugiere que cuando se de esta noticia los padres sujeten al niño entre sus brazos y tratar de explicar de forma relajada, con palabras no técnicas y claras para que así puedan asimilar y entender mucho mejor esta información otorgada ${ }^{9}$. Muchas familias tardan en aceptar la condición de su hijo, motivo por lo cual es recomendable dar este tipo de noticias conjuntamente con un profesional de psicología quien pueda solventar sus problemas psicológicos-emocionalesafectivos que conlleva el dar esta noticia.

Las personas en su mayoría niños que son diagnósticas con SD por mosaico se desarrollan de modo muy parecido al resto de niños de su edad, aunque lo hacen a una velocidad menor a la esperada ${ }^{10}$. Existen múltiples estudios en los cuales han demostrado que ciertas áreas como por ejemplo el desarrollo social es mucho más fácil para los niños con esta condición en comparación a niños sin SD, pero siendo el desarrollo motor y de aprendizaje más enlentecido que el de los niños sin este padecimiento ${ }^{10}$. 


\section{CONCLUSIÓN}

Muy aparte de las personas que presentan síndrome de Down por trisomía del cromosoma 21 que es el más común y en el cual se observan fácilmente las características clínicas tanto externas como internas de estos pacientes, las personas que presentan síndrome de Down por mosaico sus características físicas externas son poco perceptibles en una atención médica rápida ya que muchas de las veces solo presentan rasgos muy sutiles a nivel de frente, ocular, nariz, cuello y muy rara vez alteraciones como los pliegues simianos en palmas de las manos y plantas de los pies; de igual manera suelen estar acompañados estos pacientes con tono muscular conservado y discapacidad intelectual leve sin progresar a moderada a diferencia de las personas con síndrome de Down por trisomía 21.

\section{REFERENCIAS}

1.Centers for diseases Control and Prevention [Internet]. Atlanta: NCBDDD; 2014 [Cited 2020 marzo 11]. Birth defects. Available from: http:// www.cdc.gov/ ncbddd/birthdefects/downsyndrome/ data.html

2.Papavassiliou P, Charalsawadi C, Rafferty $\mathrm{K}$, Jackson Cook C. Mosaicism for trisomy 21: a review. Am J Med Genet A. 2020 Abril;167A (1):26-39. DOI 10.1002/ ajmg.a.36861.

3.Sociedad Nacional de Sindrome de Down [ Base de datos en línea]. New York; 2017. [Fecha de acceso 16 de mayo 2020]. URL disponible

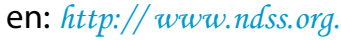

4.Giammatteo L, Kofman S, Cervantes B. Prevalencia de mosaicismo para la trisomía 21 y análisis de las variantes citogenéticas en pacientes con diagnóstico de síndrome de Down. Revisión de 24 años (1986-2010) del Servicio de Genética del Hospital General de México Dr. Eduardo Liceaga. Boletín Medico Hospital Infantil (México) [Internet]. 2013 EneFeb [consultado 2020 Abril 11];70(1):[31-6]. Disponible en: http://www.scielo.org.mx/scielo. php? pid $=S 166511462013000100007$ Q script $=$ sci arttextoting=en

5.Mohammad K, Mansoor S, Majid K. Down
Syndrome: Current Status, Challenges and Future Perspectives. International Journal of Molecular and Cellular Medicine 2016; 5 (3), $126-133$.

6.Cruz M, Bosch J. Manual llustrado de Enfermedades Raras. España: Editorial Ergon; 2015; 5 : 242 - 243.

7.Lyons K, Crandall M, Del Campo $M$. Recognizable Patterns of Human Malformation. USA: Elsevier: 2013; 1: 7 - 8.

8.Mayo Clinic. Síndrome de Down causas. [disponible en internet]. USA 2019. [fecha de acceso 17 de mayo 2020]. URL disponible en:

https://www.mayoclinic.org/es-es/diseases-conditions/ down-syndrome/symptoms-causes/syc-20355977

9.Ivan D, Cromwell P. Clinical Practice Guidelines for Management of Children With Down Syndrome: Part I. J Pediatr Health Care. 2014 Jan;28(1):105-10.

10.VV. AA. Programa Español de Salud para personas con Síndrome de Down. Ed. FEISD (Federación Española del Síndrome de Down) Madrid 2011; 89 Págs. [disponible en Internet http://www.sindromedown.net/adjuntos/ cPublicaciones/90L_downsalud.pdf con acceso 27-042020. 